\title{
Classificação da capacidade produtiva de povoamentos não desbastados de clones de eucalipto
}

\author{
Marcio Leles Romarco de Oliveira ${ }^{(1)}$ Helio Garcia Leite(2) Gilciano Saraiva Nogueira(1) $^{(1)}$ \\ Silvana Lages Ribeiro Garcia(3) $^{(3)}$ Agostinho Lopes de Souza ${ }^{(2)}$
}

\begin{abstract}
(1)Universidade Federal dos Vales do Jequitinhonha e Mucuri, Departamento de Engenharia Florestal, Rua da Glória, № 187, CEP 39100-000 Diamantina, MG. E-mail: marcioromarco@gmail.com, nogueirags@yahoo.com.br (2)Universidade Federal de Viçosa, Departamento de Engenharia Florestal, CEP 36570-000 Viçosa, MG. E-mail: hgleite@gmail.com, alsouza@ufv.br ${ }^{(3)}$ Faculdade de Viçosa, Rua Dr. Milton Bandeira, no 380, CEP 36570-000 Viçosa, MG. E-mail: garciaslr@yahoo.com.br
\end{abstract}

Resumo - O objetivo deste trabalho foi desenvolver e propor um procedimento para a estratificação de florestas de clones de eucalipto não desbastados, para a classificação de sua capacidade produtiva. Utilizaram-se dados provenientes de 70 clones, distribuídos em 5.020 parcelas permanentes de inventários florestais contínuos, com pelo menos três medições, de periodicidade anual, em cada clone. Para todos os clones, foi ajustado o modelo de Schumacher para as variáveis: área basal; altura dominante; diâmetro médio; e volume comercial, com casca. Com esses parâmetros, foi utilizado o método de Tocher, que se mostrou eficiente para agrupar clones com tendências semelhantes de crescimento em altura dominante. A estimativa de índice de local, para clones com menos de três medições, pode ser determinada a partir das informações de altura dominante, diâmetro médio, área basal e volume, obtidas do inventário florestal contínuo de outros clones.

Termos para indexação: Eucalyptus, análise de agrupamento, análise de componentes principais, índice de local, inventário florestal, método da curva-guia.

\section{Classification of the productive capacity of unthinned stands of eucalypt clones}

\begin{abstract}
The objective of this work was to develop and propose a procedure for stratification of unthinned eucalyptus clone forest, in order to classify its productive capacity. Data from 70 clones, distributed into 5,020 permanent plots of continuous forest inventories, were used with at least three annual measurements for each clone. For all clones, the Schumacher model was fit for the variables: basal area; dominant height; mean diameter; and commercial volume, with bark. The Tocher method was used to group clones displaying similar dominant height growth tendencies, and this procedure showed to be efficient. Site index estimate, for clones with less than tree measurements, can be determined from information regarding dominant height, mean diameter, basal area and commercial volume obtained from continuous forest inventories.
\end{abstract}

Index terms: Eucalyptus, cluster analysis, principal component analysis, site index, forest inventory, guide-curve method.

\section{Introdução}

As florestas clonais de eucalipto proporcionam maior uniformidade da matéria-prima florestal, melhor adaptação aos diferentes ambientes de plantio, maior produção de madeira por unidade de área, racionalização das atividades operacionais e redução na idade de corte (Santos et al., 2006). Com essas vantagens, o uso de práticas de manejo adequadas a essas florestas é fundamental para a realização de um planejamento consistente do empreendimento florestal.

O manejo de povoamentos eqüiâneos exige conhecimento sobre seus três elementos essenciais: a classificação de terras e da capacidade produtiva; prescrições; e predição de estoques de colheita (Davis \& Johnson, 1987). Portanto, a classificação da capacidade produtiva está presente em dois dos três elementos essenciais ao manejo florestal.

A capacidade produtiva de um local pode ser definida como o potencial para a produção de madeira (ou outro produto), em uma determinada área, para uma determinada espécie ou clone. Essa capacidade pode ser expressa de modo empírico (alta, média e baixa), ou por meio de índices quantitativos, como o índice de local (altura total média das árvores dominantes do

Pesq. agropec. bras., Brasília, v.43, n.11, p.1559-1567, nov. 2008 
povoamento, em uma idade específica). Além disso, a avaliação da capacidade produtiva pode ser feita por meio de métodos diretos (relação altura dominante e idade) ou indiretos, com base na vegetação indicadora e em fatores edafoclimáticos (Campos \& Leite, 2006). Conforme esses autores, a rápida expansão do uso de fertilizantes, em reflorestamentos com eucalipto, pode resultar na necessidade de revisão nos métodos de classificação usuais.

Historicamente, curvas de índices de local eram elaboradas com apenas uma espécie, para determinada região, sob determinado regime de manejo e espaçamento. Com o advento dos plantios clonais de eucalipto, tem-se a necessidade de agrupamento de clones com padrões de desenvolvimento semelhantes, sob um mesmo regime de manejo e espaçamento. Contudo, em muitos casos, o agrupamento de clones não é suficiente para o propósito de classificação, em razão da dinâmica de entrada e saída dos clones nesses plantios, em que, a cada ano, eles são substituídos por materiais genéticos com maior potencial produtivo. Conforme Heger (1973), a idade-índice considerada, o tipo de habitat ou ecorregião, a composição dos dados utilizados para a construção das curvas e a presença de diferentes materiais genéticos (clones) resultam em feixes de curvas com diferentes formas. Por alguns desses motivos, muitas vezes, o desejável balanceamento quanto à freqüência dos índices de local, encontrados nas diferentes classes de produtividade, não é verificado (Campos \& Leite, 2006).

Existem vários estudos sobre a classificação da capacidade produtiva, mediante a construção de curvas de índice de local. Nesses estudos, é possível verificar que, na maioria das vezes, as classificações são feitas pelo método da curva-guia, com dados de parcelas permanentes ou de análise de tronco. Contudo, são raros os estudos sobre plantios clonais de eucalipto em regiões tropicais, entre eles o de Tonini et al. (2004), que contemplaram casos em que o número de parcelas permanentes e de medições delas foi suficiente para a construção de curvas específicas por clone.

O índice de local é uma variável importante em estudos de crescimento e produção, e qualquer distorção na determinação deste índice influencia as estimativas de produção. Estimativas consistentes somente podem ser obtidas com uma avaliação precisa do potencial de crescimento (Parresol \& Vissage, 1998).

Com essas considerações e a significativa ocorrência de plantios de clones de eucalipto no Brasil, este estudo foi realizado com o objetivo de desenvolver um procedimento para a estratificação de florestas de clones de eucalipto e sua classificação quanto à capacidade produtiva.

\section{Material e Métodos}

Os dados utilizados no presente trabalho são provenientes de inventários florestais contínuos, realizados entre os anos de 1996 e 2006, em povoamentos de clones de eucalipto no Estado da Bahia, que compreendiam cerca de 70 mil ha de florestas.

Inicialmente, foram utilizados dados de 70 clones, distribuídos em 5.020 parcelas permanentes, e cada clone tinha pelo menos três medições, com periodicidade anual.

Em todas as parcelas mensuradas, foram determinados, anualmente, a altura dominante média ( $\mathrm{Hd})$; a área basal por hectare (B); o diâmetro médio (q); e o volume comercial com casca por hectare (V). Os dados foram submetidos à criteriosa análise de consistência, conforme recomendações de Curtis \& Marshall (2005) e Campos \& Leite (2006); alguns dados foram eliminados, por se tratarem de "outliers" (Draper \& Smith, 1998), enquanto outros foram eliminados para tornar compatível a freqüência de parcelas permanentes, de acordo com as condições de produtividade encontradas nos povoamentos.

Para cada um dos 70 clones em escala comercial, com mais de três medições de parcelas permanentes, foi ajustada a função de crescimento de Schumacher (1939), na forma linear, para as seguintes variáveis dependentes: área basal (B), altura dominante (Hd), diâmetro médio (q) e volume comercial com casca (V), no total de oito estimativas de parâmetros para cada clone. A relação funcional utilizada foi: Ln $\mathrm{Y}=\beta_{0}+\beta_{1} \mathrm{I}^{-1}+\varepsilon$, em que: $\mathrm{Y}$ é a variável dependente; $\beta_{0}$ e $\beta_{1}$ são parâmetros da regressão; I, a idade, em meses; $\varepsilon$, o erro aleatório, com: $\varepsilon \sim \mathrm{N}\left(0, \sigma^{2}\right)$.

Com o propósito de estratificar os clones por grupos semelhantes em crescimento, foi empregada a análise de agrupamento com base nos oito parâmetros $\hat{\beta}_{0}$ e $\hat{\beta}_{1}$, estimados para a totalidade dos clones. Essas variáveis foram utilizadas por serem relacionadas à produtividade de povoamento e à qualidade do local. 
A análise de agrupamento é uma técnica multivariada, amplamente utilizada em ciência florestal para diversos fins (Martins et al., 2003; Santos et al., 2004; Albuquerque et al., 2006). O processo de agrupamento envolve a estimação de uma medida de similaridade (ou dissimilaridade) e a adoção de uma técnica de agrupamento para a formação dos grupos (Cruz et al., 2004). No presente trabalho, foram utilizados: a distância euclidiana média; a área basal; a altura dominante; e o diâmetro médio (Abreu et al., 2002). O método de agrupamento utilizado foi o de Tocher.

Após a obtenção do primeiro agrupamento, foi realizada a análise de componentes principais, com o objetivo de se descartarem parâmetros irrelevantes. A variável com maior coeficiente de ponderação (autovetor), no componente de menor autovalor, foi considerada de menor importância para explicar a variabilidade do material estudado, e passível de descarte. O propósito foi verificar a possibilidade de exclusão ou não de algum parâmetro da regressão e, em conseqüência, de alguma variável mensurada.

Com a determinação do parâmetro de menor importância, o agrupamento foi realizado novamente e comparado com o formado por todas as variáveis; quando o novo agrupamento era igual ao agrupamento com todas variáveis, esse parâmetro era descartado. $\mathrm{O}$ processo foi repetido até que fosse obtida a alteração do agrupamento, quando comparado ao de todas as variáveis. Quando ocorreu diferença entre os agrupamentos, a variável foi mantida e o processo de agrupamento foi encerrado.

Após a etapa de descarte de variável, usou-se o agrupamento final (método de Tocher) com as variáveis selecionadas. Esseagrupamento foirealizado sucessivamente até ter-se um número de estratos que agrupasse os clones com tendências de crescimento semelhantes.

O método para determinação dos índices de local foi o da curva-guia (Dias et al., 2005b), utilizado por ser compatível com as características dos dados em foco e por sua simplicidade e eficiência. Cabe reportar que este método é o mais difundido entre as empresas florestais no Brasil (Campos \& Leite, 2006).

O modelo logístico (Draper \& Smith, 1998), foi ajustado para cada estrato: $\mathrm{Hd}=\beta_{0}\left(1+\beta_{1} \mathrm{e}^{-\beta_{2} \mathrm{I}}\right)^{-1} \varepsilon$, em que: Hd é a altura dominante; $\beta_{0}, \beta_{1}$ e $\beta_{2}$ são os parâmetros da função; I é a idade; e $\varepsilon$ é o erro aleatório, sendo $\varepsilon \sim N\left(0, \sigma^{2}\right)$.
Além dos 70 clones com mais de três medições em parcelas permanentes, foram analisados 6 clones que possuíam apenas uma medição e mais 2 clones com duas medições. Para os clones com uma só medição, foram utilizadas informações da idade, altura dominante, área basal, volume comercial e diâmetro médio.

Com o uso da função de crescimento de Schumacher ajustada para cada clone, foram estimados os valores de Hd, V, B e q, para uma mesma idade específica do clone analisado. Estimadas as variáveis para cada clone, foi empregada a distância euclidiana média (Cruz et al., 2004), para se calcular a similaridade entre as estimativas de $\mathrm{Hd}, \mathrm{V}, \mathrm{B}$ e q, dos clones com menos de três medições.

Em seguida, foi determinado a qual clone - com mais de três medições - o clone analisado era mais similar, isto foi feito com base na menor distância verificada.

Em seguida, após a determinação do clone com maior similaridade, foi verificado a qual estrato o clone analisado pertencia. Isso permitiu empregar a equação de local do estrato mais similar, na determinação do seu índice de local.

Para se verificar a necessidade da estratificação dos diferentes clones, para definição dos índices de local para estudos de crescimento e produção, foram avaliadas duas alternativas, com uso do modelo de Clutter et al. (1963):

$$
\left\{\begin{array}{l}
\operatorname{Ln} B_{2}=\operatorname{Ln} B_{1}\left(\frac{I_{1}}{I_{2}}\right)+\alpha_{1}\left(1-\frac{I_{1}}{I_{2}}\right)+\alpha_{2}\left(1-\frac{I_{1}}{I_{2}}\right) S+\varepsilon \\
\operatorname{Ln} V_{2}=\beta_{0}+\beta_{1} \frac{1}{I_{2}}+\beta_{2} S+\beta_{3} \operatorname{Ln} B_{2}+\varepsilon
\end{array}\right.
$$

em que: $\mathrm{I}_{1}$ é a idade atual; $\mathrm{I}_{2}$ é a idade futura; $\mathrm{B}_{1}$ é a área basal na idade $\mathrm{I}_{1} ; \mathrm{B}_{2}$ é a área basal na idade $\mathrm{I}_{2} ; \mathrm{S}$ é o índice de local; $\mathrm{V}_{2}$ é o volume; $\mathrm{Ln}$ é o logaritmo neperiano; $\beta_{0}, \beta_{1}, \beta_{2}, \beta_{3}, \alpha_{1}$ e $\alpha_{2}$ são parâmetros do modelo; e $\varepsilon$ é o erro aleatório, em que $\varepsilon \sim N\left(0, \sigma^{2}\right)$.

$\mathrm{Na}$ primeira alternativa, ajustou-se o modelo de Clutter com o uso das estimativas de índices de local, obtidas por meio de uma equação única, sem estratificação dos clones. Na segunda alternativa, ajustou-se o modelo de Clutter, para cada estrato, pelo uso das estimativas de índices de local, obtidas das equações específicas de cada estrato. 
A partir do sistema de equações, correspondentes a cada uma dessas duas alternativas, foi projetado o volume de 2005 para 2006, e suas estimativas foram comparadas com os volumes observados em 2006.

\section{Resultados e Discussão}

Com as estimativas dos parâmetros do modelo de Schumacher linearizado, $\mathrm{LnY}=\beta_{0}+\beta_{1} \mathrm{I}^{-1}+\varepsilon$, obtidas para cada clone e para cada variável mensurada, efetuou-se a análise de agrupamento pelo método de Tocher, com base na distância euclidiana média, o que teve como resultado três grupos. $\mathrm{O}$ grupo 1 foi constituído por 1 clone (68), o grupo 2 por 2 (28 e 31 ) e o grupo 3 por 67 clones.

Após a formação dos grupos, procedeu-se à análise de componentes principais, para a verificação dos parâmetros que apresentavam a menor importância, e que seriam passíveis de descarte (Tabela 1). O parâmetro que menos contribuiu foi a inclinação $\left(\beta_{1}\right)$ da variável volume, por apresentar o maior valor absoluto de autovetor no último componente formado; o último componente apresentou menor autovalor na comparação entre os demais.

Com a exclusão da variável $\beta_{1} \_$V, o agrupamento foi realizado novamente. Observou-se que houve alteração no agrupamento do clone 31 , que estava no grupo 2 e que passou a fazer parte do grupo 3. Os demais permaneceram no mesmo grupo (Tabela 2). Entretanto, a variável eliminada $\left(\beta_{1}\right.$ V) foi importante para a definição dos grupos e não pode ser desconsiderada.
Com o entendimento de que todos os parâmetros são importantes para o agrupamento, o grupo 3, da Tabela 2, foi submetido a um novo reagrupamento (agrupamento 1). Com isso, foram definidos mais 7 novos grupos, em que o último foi formado por 46 clones, representados pelo agrupamento $2 \mathrm{da}$ Tabela 3.

Para esse grupo, representado pelos 46 clones, foram realizadas análises gráficas e observadas tendências diferentes de crescimento em altura dominante. Assim, decidiu-se por reagrupar novamente os 46 clones. Como conseqüência, formaram-se mais 13 grupos, representados pelo agrupamento 2. Desta forma, os 70 clones foram finalmente agrupados em 25 grupos (Tabela 3).

As equações estimadas para os estratos com mais de três medições foram relacionadas e apresentaram coeficientes de correlação superiores a $87 \%$ (Tabela 4).

Com os coeficientes da equação, para cada estrato foi estimada a altura dominante com idade de 24 a 96 meses (Figura 1). Observou-se, na maioria dos estratos, crescimento em altura dominante após 96 meses. O grupo 2 apresentou estagnação do crescimento em altura dominante por volta de 60 meses. Todos os estratos apresentaram tendências de crescimento distintas, o que sinaliza a importância da estratificação, para se classificar a capacidade produtiva.

Em alguns casos, essa estratificação pode ser feita com base em outros atributos, para aumento da precisão,

Tabela 1. Valores dos autovetores dos componentes principais analisados.

\begin{tabular}{|c|c|c|c|c|c|c|c|c|}
\hline \multirow[t]{2}{*}{ Parâmetro } & \multicolumn{8}{|c|}{ Componentes } \\
\hline & 1 & 2 & 3 & 4 & 5 & 6 & 7 & 8 \\
\hline$\beta_{0 \_} \mathrm{V}$ & $-0,3017$ & $-0,4972$ & $-0,1647$ & 0,6374 & $-0,0419$ & $-0,0388$ & 0,2080 & 0,4265 \\
\hline$\beta_{1-} \mathrm{V}$ & 0,4287 & $-0,1441$ & 0,2592 & $-0,3814$ & 0,1616 & $-0,1794$ & 0,2879 & 0,6646 \\
\hline$\beta_{0}-\mathrm{B}$ & $-0,3542$ & $-0,1872$ & 0,4785 & $-0,0434$ & 0,6942 & 0,1697 & 0,2057 & $-0,2358$ \\
\hline$\beta_{1 \_} \mathrm{B}$ & 0,3939 & $-0,3740$ & $-0,1726$ & $-0,0558$ & $-0,1845$ & 0,3610 & 0,5827 & $-0,4100$ \\
\hline$\beta_{0}-\mathrm{Hd}$ & $-0,2748$ & $-0,4409$ & $-0,4539$ & $-0,4909$ & 0,1049 & $-0,4940$ & $-0,0516$ & $-0,1594$ \\
\hline$\beta_{1}-\mathrm{Hd}$ & 0,3868 & $-0,2193$ & 0,3975 & 0,3510 & $-0,0438$ & $-0,6162$ & $-0,1295$ & $-0,3503$ \\
\hline$\beta_{0 \_} \mathrm{q}$ & $-0,2749$ & $-0,3933$ & 0,5054 & $-0,2785$ & $-0,5823$ & 0,2063 & $-0,2324$ & 0,0331 \\
\hline$\beta_{1} \_\mathrm{q}$ & 0,3793 & $-0,3999$ & $-0,1659$ & 0,0401 & 0,3226 & 0,3754 & $-0,6470$ & 0,0595 \\
\hline
\end{tabular}

Tabela 2. Agrupamento dos 70 clones de eucalipto, após a eliminação do parâmetro $\beta 1$ V.

\begin{tabular}{|c|c|c|c|c|c|c|c|c|c|c|c|c|c|c|c|c|c|}
\hline Grupos & \multicolumn{17}{|c|}{ Clones } \\
\hline 1 & 68 & & & & & & & & & & & & & & & & \\
\hline 2 & 28 & & & & & & & & & & & & & & & & \\
\hline 3 & 1 & 2 & 3 & 4 & 5 & 6 & 7 & 8 & 9 & 10 & 11 & 12 & 13 & 14 & 15 & 16 & 17 \\
\hline & 18 & 19 & 20 & 21 & 22 & 23 & 24 & 25 & 26 & 27 & 29 & 30 & 31 & 32 & 33 & 34 & 35 \\
\hline & 36 & 37 & 38 & 39 & 40 & 41 & 42 & 43 & 44 & 45 & 46 & 47 & 48 & 49 & 50 & 51 & 52 \\
\hline & 53 & 54 & 55 & 56 & 57 & 58 & 59 & 60 & 61 & 62 & 63 & 64 & 65 & 66 & 67 & 69 & 70 \\
\hline
\end{tabular}


conforme Campos \& Leite (2006). Alguns clones apresentaram tendência mais suave de crescimento, ao longo do tempo, enquanto outros tiveram crescimento inicial maior, mas chegaram à estagnação mais cedo.

No Tabela 5, são apresentadas: as médias das variáveis observadas $\mathrm{V}, \mathrm{B}, \mathrm{Hd}$ e q, para os clones com menos de três medições (clones 71 a 78); as estimativas dessas variáveis, obtidas pela equação de Schumacher, para os clones com mais de três medições, quanto à idade do clone analisado; e a distância euclidiana média.

De modo geral, quanto maior a distância euclidiana média, maior foi a diferenciação entre as variáveis dos clones (Tabela 5). No entanto, os clones 72 e 29 resultaram em menor diferença entre as variáveis analisadas.
Com a definição do estrato para os clones com menos de três medições, foi possível estimar-se o índice de local em qualquer idade, para fins de ajustes de modelos de crescimento e produção.

Para se avaliar a utilidade da estratificação adotada, foi ajustado o modelo de Clutter para oito dos 25 grupos de índices de local definidos. A análise prévia indicou que, para apenas oito estratos, foi possível ajustar o modelo de Clutter, em razão de não se ter a limitação da freqüência de dados e, também, por ocorrer uma tendência definida de volume ao longo do tempo. Na Tabela 6 , foram relacionadas as equações e estatísticas, para cada estrato e para o agrupamento total. Foi retirada a variável $\left[\left(1-\left(\mathrm{I}_{1} / \mathrm{I}_{2}\right)\right] \mathrm{S}\right.$ da expressão da área basal, por não ser significativa estatisticamente,

Tabela 3. Reagrupamento dos 67 clones, inicialmente pertencentes ao grupo 3 da Tabela 2.

\begin{tabular}{|c|c|c|c|c|c|c|c|c|c|c|c|}
\hline Agrupamento & Grupos & \multicolumn{10}{|c|}{ Clones } \\
\hline \multirow[t]{7}{*}{1} & 3 & 20 & 32 & 35 & 36 & 41 & 46 & 47 & 63 & 68 & \\
\hline & 4 & 3 & 7 & & & & & & & & \\
\hline & 5 & 50 & 53 & 54 & 67 & & & & & & \\
\hline & 6 & 10 & 21 & 26 & & & & & & & \\
\hline & 7 & 59 & 66 & & & & & & & & \\
\hline & 8 & 24 & & & & & & & & & \\
\hline & 9 & 69 & & & & & & & & & \\
\hline \multirow[t]{16}{*}{2} & 10 & 1 & 4 & 9 & 12 & 14 & 15 & 25 & 58 & & \\
\hline & 11 & 8 & 30 & 52 & 57 & & & & & & \\
\hline & 12 & 5 & 6 & 13 & 29 & 33 & 34 & 37 & 38 & 42 & 64 \\
\hline & 13 & 2 & 27 & 62 & 65 & & & & & & \\
\hline & 14 & 40 & 45 & & & & & & & & \\
\hline & 15 & 16 & 51 & 60 & & & & & & & \\
\hline & 16 & 17 & 49 & & & & & & & & \\
\hline & 17 & 18 & 43 & 70 & & & & & & & \\
\hline & 18 & 39 & 48 & & & & & & & & \\
\hline & 19 & 11 & 55 & & & & & & & & \\
\hline & 20 & 56 & & & & & & & & & \\
\hline & 21 & 61 & & & & & & & & & \\
\hline & 22 & 23 & & & & & & & & & \\
\hline & 23 & 19 & & & & & & & & & \\
\hline & 24 & 44 & & & & & & & & & \\
\hline & 25 & 22 & & & & & & & & & \\
\hline
\end{tabular}

Tabela 4. Resultado do ajuste do modelo logístico, a partir da estratificação final dos clones em 25 grupos.

\begin{tabular}{|c|c|c|c|c|c|c|c|c|c|}
\hline Estrato & $\hat{\beta}_{0}$ & $\hat{\beta}_{1}$ & $\hat{\beta}_{2}$ & $\mathrm{r}_{\hat{y} \mathrm{y}}$ & Estrato & $\hat{\beta}_{0}$ & $\hat{\beta}_{1}$ & $\hat{\beta}_{2}$ & $r_{\hat{y} y}$ \\
\hline 1 & 36,1642 & 3,6825 & 0,0326 & 0,9544 & 14 & 32,4823 & 3,6431 & 0,0505 & 0,9234 \\
\hline 2 & 28,0682 & 12,4064 & 0,1197 & 0,8713 & 15 & 35,0507 & 3,9709 & 0,0563 & 0,9763 \\
\hline 3 & 35,3290 & 3,1154 & 0,0368 & 0,9402 & 16 & 35,6909 & 3,6999 & 0,0533 & 0,9851 \\
\hline 4 & 35,2815 & 3,7138 & 0,0468 & 0,9528 & 17 & 31,7811 & 4,1867 & 0,0556 & 0,9276 \\
\hline 5 & 34,2375 & 6,2016 & 0,0749 & 0,9686 & 18 & 33,3203 & 4,8033 & 0,0622 & 0,9255 \\
\hline 6 & 34,9657 & 3,0104 & 0,0447 & 0,9702 & 19 & 36,0607 & 3,4454 & 0,0498 & 0,9457 \\
\hline 7 & 36,5625 & 6,6079 & 0,0587 & 0,9659 & 20 & 31,3078 & 6,3101 & 0,0865 & 0,9195 \\
\hline 8 & 31,6897 & 2,6274 & 0,0444 & 0,9174 & 21 & 32,1293 & 3,3075 & 0,0559 & 0,9910 \\
\hline 9 & 46,4111 & 3,9835 & 0,0300 & 0,9681 & 22 & 37,4587 & 4,0763 & 0,0518 & 0,9837 \\
\hline 10 & 33,8308 & 3,4488 & 0,0539 & 0,9320 & 23 & 37,2981 & 2,7236 & 0,0352 & 0,9590 \\
\hline 11 & 35,3701 & 3,4422 & 0,0498 & 0,9352 & 24 & 36,8987 & 4,2485 & 0,0597 & 0,9963 \\
\hline 12 & 35,1587 & 3,5889 & 0,0475 & 0,9402 & 25 & 35,1094 & 2,6570 & 0,0477 & 0,9955 \\
\hline 13 & 37,0422 & 3,2784 & 0,0459 & 0,9129 & & & & & \\
\hline
\end{tabular}



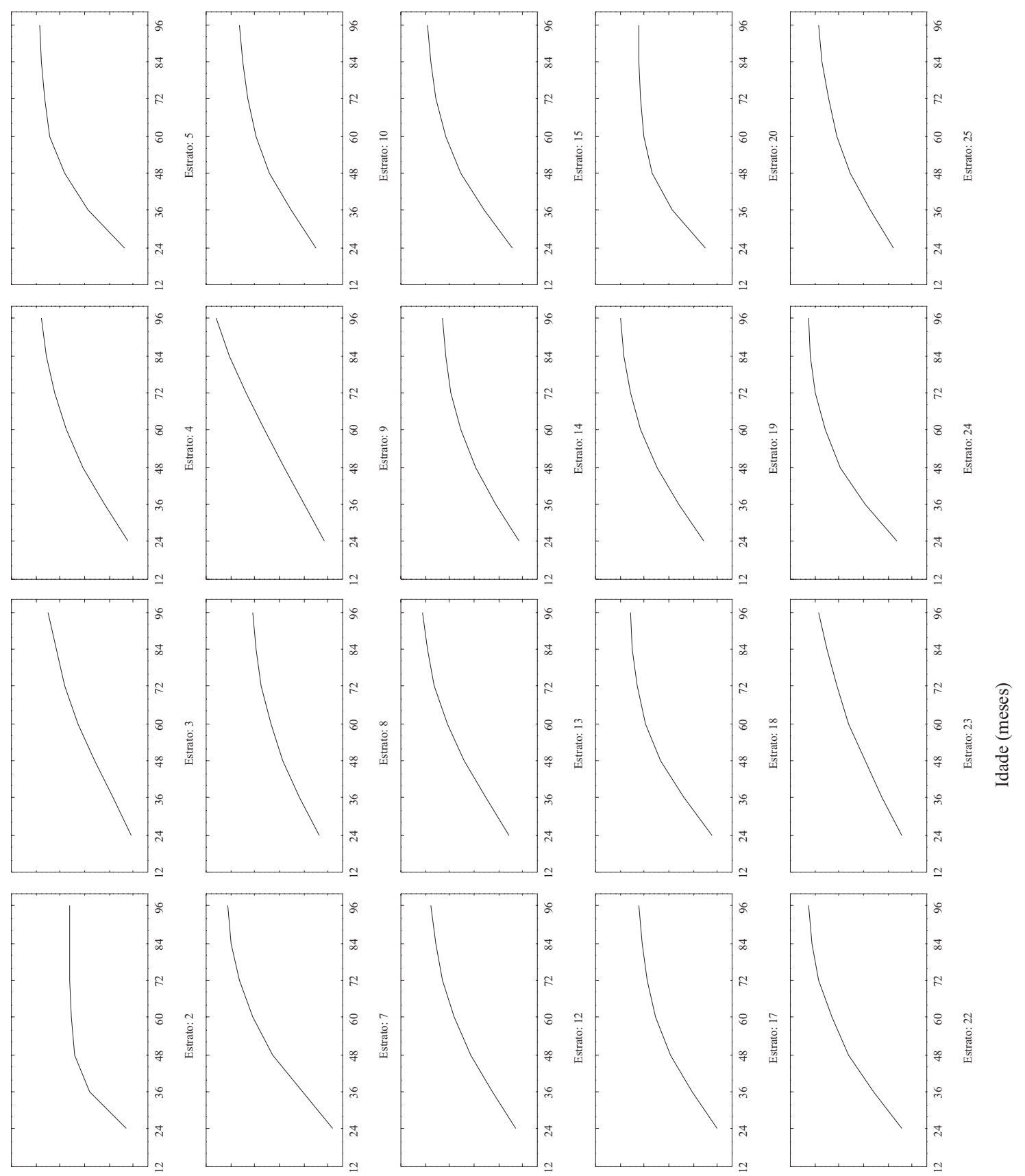

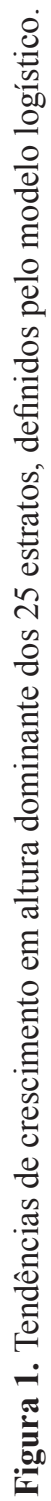

Pesq. agropec. bras., Brasília, v.43, n.11, p.1559-1567, nov. 2008 
pelo teste t, a 95\% de probabilidade. Resultados semelhantes foram encontrados por Dias et al. (2005a) e Valdez-Lazalde \& Lynch (2000).

$\mathrm{Na}$ comparação entre os valores correspondentes, observados e estimados para os modelos, observouse erro médio percentual menor que $5 \%$, em todos os sistemas. As correlações entre os valores observados e estimados, tanto para volume quanto área basal, foram maiores do que 0,80 (Tabela 6 ).
A estimativa de estoque volumétrico, realizada com e sem a estratificação proposta com os dados de 2005 para 2006, mostrou que a estratificação apresentou estoque total mais próximo do observado em 2006 (Tabela 7). As diferenças percentuais dos estoques estimados, em relação aos observados em 2006, foram de $-8,32$ e $-6,62 \%$, respectivamente.

A Figura 2 ilustra as estimativas de incremento médio anual aos sete anos (IMA7), realizadas com os dados de 2006 (última medição do inventário florestal

Tabela 5. Estimativa média das variáveis volume (V), área basal (B), altura dominante (Hd) e diâmetro médio (q) para uma mesma idade, com uso dos parâmetros do modelo de Schumacher e distância euclidiana média.

\begin{tabular}{|c|c|c|c|c|c|c|c|}
\hline Estrato & Clone & Idade & $\mathrm{V}\left(\mathrm{m}^{3} \mathrm{ha}^{-1}\right)$ & $\mathrm{B}\left(\mathrm{m}^{2} \mathrm{ha}^{-1}\right)$ & $\mathrm{Hd}(\mathrm{m})$ & $\mathrm{q}(\mathrm{cm})$ & Distância euclidiana média \\
\hline \multirow[t]{2}{*}{5} & 71 & 23 & 87,29 & 12,02 & 16,63 & 12,74 & 0,5576 \\
\hline & 54 & & 75,82 & 13,06 & 16,39 & 13,54 & \\
\hline Igualdade $(\%)^{(1)}$ & & & 86,86 & 108,67 & 98,57 & 106,25 & \\
\hline \multirow[t]{2}{*}{12} & 72 & 23 & 47,71 & 8,31 & 14,00 & 11,29 & 0,1029 \\
\hline & 29 & & 45,56 & 8,31 & 14,26 & 11,26 & \\
\hline Igualdade (\%) & & & 95,50 & 100,10 & 101,84 & 99,75 & \\
\hline \multirow[t]{2}{*}{4} & 73 & 26 & 69,51 & 8,75 & 17,75 & 12,71 & 0,3110 \\
\hline & 3 & & 63,72 & 9,75 & 17,62 & 12,57 & \\
\hline Igualdade (\%) & & & 91,68 & 111,47 & 99,29 & 98,87 & \\
\hline \multirow[t]{2}{*}{19} & 74 & 26 & 98,51 & 11,81 & 18,25 & 13,28 & 0,2349 \\
\hline & 55 & & 98,13 & 11,60 & 18,79 & 13,74 & \\
\hline Igualdade (\%) & & & 99,61 & 98,21 & 102,93 & 103,46 & \\
\hline \multirow[t]{2}{*}{3} & 75 & 23 & 32,92 & 6,77 & 13,25 & 10,07 & 0,1419 \\
\hline & 63 & & 32,25 & 6,69 & 13,41 & 10,43 & \\
\hline Igualdade (\%) & & & 97,97 & 98,88 & 101,17 & 103,57 & \\
\hline \multirow[t]{2}{*}{19} & 76 & 23 & 77,52 & 10,47 & 16,25 & 12,19 & 0,3008 \\
\hline & 55 & & 73,87 & 9,83 & 16,60 & 12,70 & \\
\hline Igualdade (\%) & & & 95,30 & 93,93 & 102,16 & 104,21 & \\
\hline \multirow[t]{2}{*}{12} & 77 & 60 & 219,49 & 16,60 & 31,00 & 22,05 & 1,7025 \\
\hline & 38 & & 303,24 & 22,76 & 31,32 & 19,59 & \\
\hline Igualdade (\%) & & & 126,59 & 110,78 & 115,60 & 112,72 & \\
\hline \multirow[t]{2}{*}{1} & 78 & 62 & 278,63 & 17,45 & 25,08 & 14,40 & 0,6875 \\
\hline & 31 & & 188,71 & 18,79 & 25,16 & 15,11 & \\
\hline Igualdade (\%) & & & 67,73 & 107,66 & 100,31 & 104,95 & \\
\hline
\end{tabular}

${ }^{(1)}$ Igualdade percentual entre a variável do clone, com uma ou duas medições, e a do clone com mais de três medições.

Tabela 6. Estratos de índice de local, equação ajustada do modelo de Clutter, para as duas alternativas utilizadas, e seus coeficientes estatísticos.

\begin{tabular}{|c|c|c|c|c|c|c|c|c|c|}
\hline \multirow[t]{2}{*}{ Estrato } & \multicolumn{3}{|c|}{ Alternativa 1} & \multicolumn{6}{|c|}{ Alternativa 2} \\
\hline & $\hat{\alpha}_{1}$ & $\mathrm{R}^{2}$ & $\varepsilon(\%)$ & $\hat{\beta}_{0}$ & $\hat{\beta_{1}}$ & $\hat{\beta}_{2}$ & $\hat{\beta_{3}}$ & $\mathrm{R}^{2}$ & $\varepsilon(\%)$ \\
\hline 3 & 3,645130 & 0,96 & 0,0269 & 1,619595 & $-16,82719$ & 0,012602 & 1,229073 & 0,95 & $-0,0039$ \\
\hline 10 & 3,554240 & 0,96 & $-0,0830$ & 1,802969 & $-18,27678$ & 0,013046 & 1,197233 & 0,91 & $-0,1578$ \\
\hline 11 & 3,535802 & 0,95 & 0,0902 & 0,615441 & $-11,90619$ & 0,013352 & 1,602747 & 0,89 & $-0,1113$ \\
\hline 12 & 3,699858 & 0,95 & $-0,0123$ & 2,091604 & $-23,63967$ & 0,016390 & 1,080649 & 0,93 & $-0,0110$ \\
\hline 13 & 3,491133 & 0,93 & $-0,1584$ & 1,272734 & $-17,94524$ & 0,016857 & 1,335592 & 0,84 & $-0,2891$ \\
\hline 14 & 3,589798 & 0,95 & 0,1207 & 2,317664 & $-32,65088$ & 0,015732 & 1,065577 & 0,89 & 0,1689 \\
\hline 17 & 3,568525 & 0,95 & $-0,1354$ & 1,457337 & $-20,78508$ & 0,018171 & 1,273920 & 0,86 & 0,1791 \\
\hline 19 & 3,656557 & 0,95 & $-0,0063$ & 2,122243 & $-24,74528$ & 0,014598 & 1,100748 & 0,90 & $-0,0690$ \\
\hline Geral & 3,628970 & 0,94 & $-0,0096$ & 1,904706 & $-20,57623$ & 0,017744 & 1,118232 & 0,93 & $-0,6050$ \\
\hline
\end{tabular}

${ }^{(1)}$ Equação utilizada na alternativa $1: \operatorname{Ln} B_{2}=\operatorname{Ln} B_{1}\left(\frac{I_{1}}{I_{2}}\right)+\alpha_{1}\left(1-\frac{I_{1}}{I_{2}}\right)$, em que: $I_{1}$, idade atual, em meses; $I_{2}$, idade futura, em meses; $\beta_{1}$, área basal na idade

$\mathrm{I}_{1}$, em $\mathrm{m}^{2}$ ha $^{-1} ; \beta_{2}$, área basal na idade $\mathrm{I}_{2}$, em $\mathrm{m}^{2}$ ha ${ }^{-1} ; \mathrm{S}$, índice de local, em $\mathrm{m} ; \mathrm{V}_{2}$, volume em $\mathrm{m}^{3}$ ha ${ }^{-1}$; Ln, logaritmo neperiano; equação utilizada na alternativa 2: $\operatorname{Ln} V_{2}=\beta_{0}+\beta_{1} \frac{1}{I_{2}}+\beta_{2} S+\beta_{3} \operatorname{Ln} B_{2}$, em que: $\beta_{0}$. $\beta_{1}$. $\beta_{2}, \beta_{3}, \alpha_{1}$ e $\alpha_{2}$ são parâmetros do modelo; e $\varepsilon$ é o erro aleatório, em que $\varepsilon \sim \mathrm{N}\left(0, \sigma^{2}\right)$. 
contínuo). Foi observado que, para a maioria dos estratos, o IMA7 foi muito próximo ou semelhante, e que a maior diferença ocorreu no estrato 11 . Os estratos 10 e 12 foram semelhantes e ocuparam as maiores áreas (Tabela 7). A expectativa de IMA7 é importante em todos os níveis de planejamento da maioria das empresas florestais. Cabe observar que uma diferença de $10 \%$ no IMA7 pode resultar em sérios equívocos no planejamento. Por exemplo, com a expectativa de IMA7 de $50 \mathrm{~m}^{3} \mathrm{ha}^{-1}$ por ano, mas apenas $45 \mathrm{~m}^{3} \mathrm{ha}^{-1}$ por ano observados, tem-se um deficit de área de corte anual de 1.587,3 ha, para o atendimento da demanda de 5 milhões $\mathrm{m}^{3}$.

As estimativas de volume, projetadas de 2005 para 2006, foram semelhantes na maioria das idades, nas duas alternativas de projeção (Figura 3). A exceção foi o intervalo de idade

Tabela 7. Área total (ha) e estoque de volume observado $\left(\mathrm{EVO}, \mathrm{m}^{3} \mathrm{ha}^{-1}\right)$, em 2006 , e projeções dos estoques de volumes estimados (EVE, $\mathrm{m}^{3} \mathrm{ha}^{-1}$ ) de 2005 para 2006, com o uso das duas alternativas do modelo de Clutter: sem estratificação e com estratificação, para fins de índice de local.

\begin{tabular}{crrrr}
\hline Estrato local & Área & \multirow{2}{*}{ EVO } & \multicolumn{2}{c}{ EVE } \\
\cline { 3 - 5 } & & & Alternativa 1 & Alternativa 2 \\
\hline 3 & 1.063 & 20.034 & 17.454 & 16.408 \\
10 & 23.694 & 264.700 & 244.125 & 250.505 \\
11 & 4.997 & 70.492 & 49.270 & 59.359 \\
12 & 26.131 & 311.315 & 296.280 & 291.592 \\
13 & 3.356 & 32.024 & 30.416 & 31.635 \\
14 & 364 & 6.431 & 5.546 & 5.801 \\
17 & 1.395 & 23.201 & 21.168 & 22.448 \\
19 & 3.918 & 49.198 & 48.476 & 48.205 \\
\hline Total & 64.919 & 777.395 & 712.734 & 725.952 \\
\hline
\end{tabular}

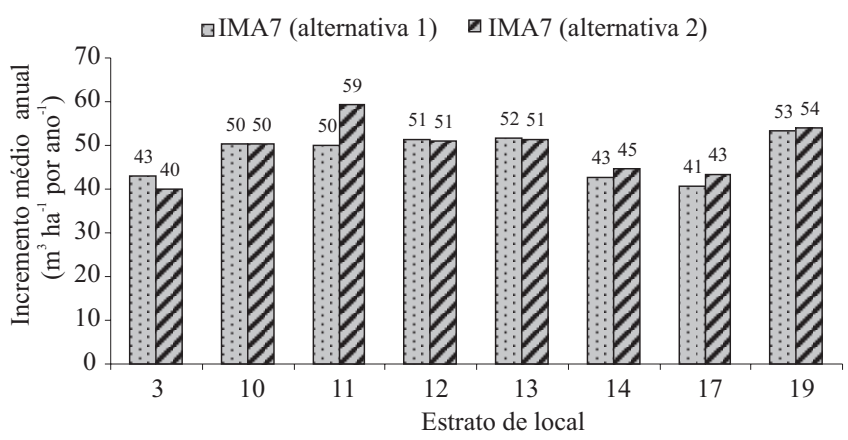

Figura 2. Incremento médio anual em volume, para a idade de sete anos, a partir dos dados de 2006, para as duas alternativas de avaliação. de 50 e 80 meses. Este fato também pode gerar problemas no planejamento do corte, já que a rotação é por volta de 72 meses, o que significa que a estratificação de capacidade produtiva é importante para as estimativas de crescimento e produção.

Vanclay et al. (1995) afirmaram que, na dúvida sobre a eficiência da estimativa de índice de local, é prudente estratificar-se a floresta pelo uso de algum fator como topografia, geologia, nível de produção ou a própria produção volumétrica.

Alguns estratos, muitas vezes, têm comportamentos semelhantes, o que torna possível seu agrupamento (Figura 4). Entretanto, melhor resultado foi obtido com a estratificação proposta. Na Figura 4, um único feixe poderia ser usado para alguns estratos, porém, com prejuízos em termos de precisão, que somente seriam quantificados com o ajuste e a aplicação de modelos de crescimento e produção.
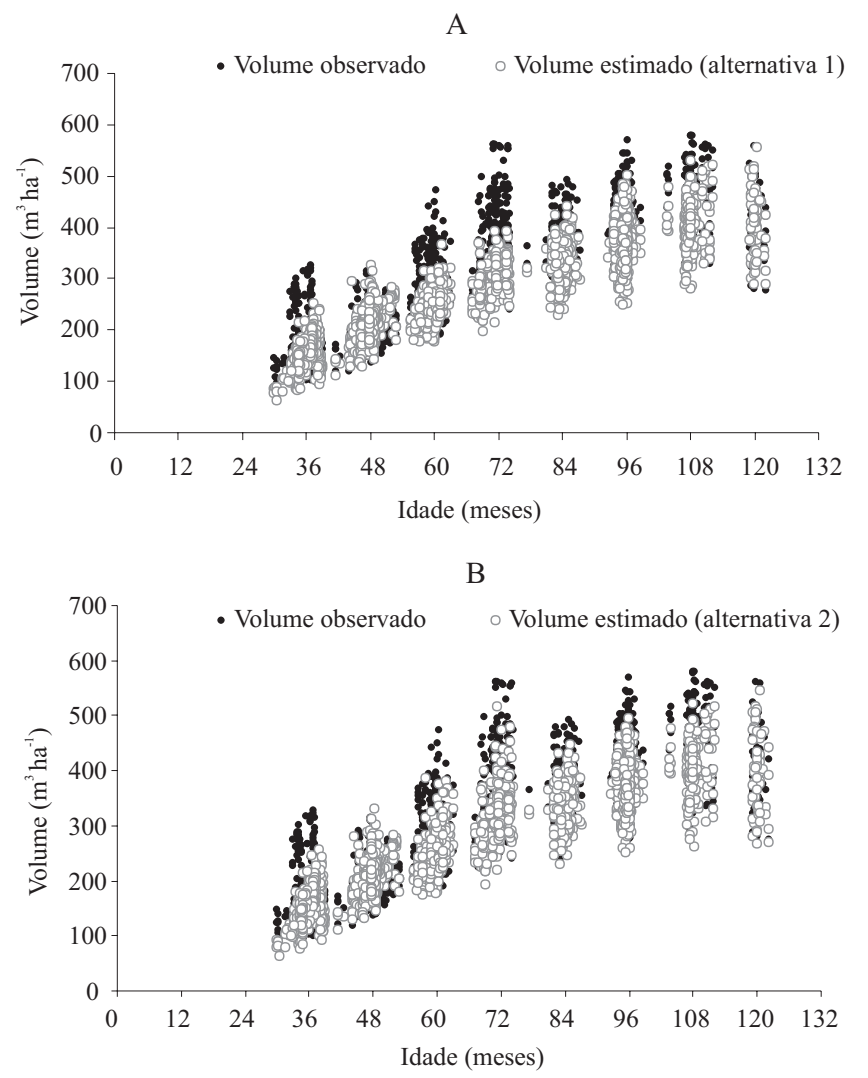

Figura 3. Volume observado em 2006, e estimativas de volume de 2005 para 2006, com a alternativa 1 (A) e a alternativa 2 (B). 


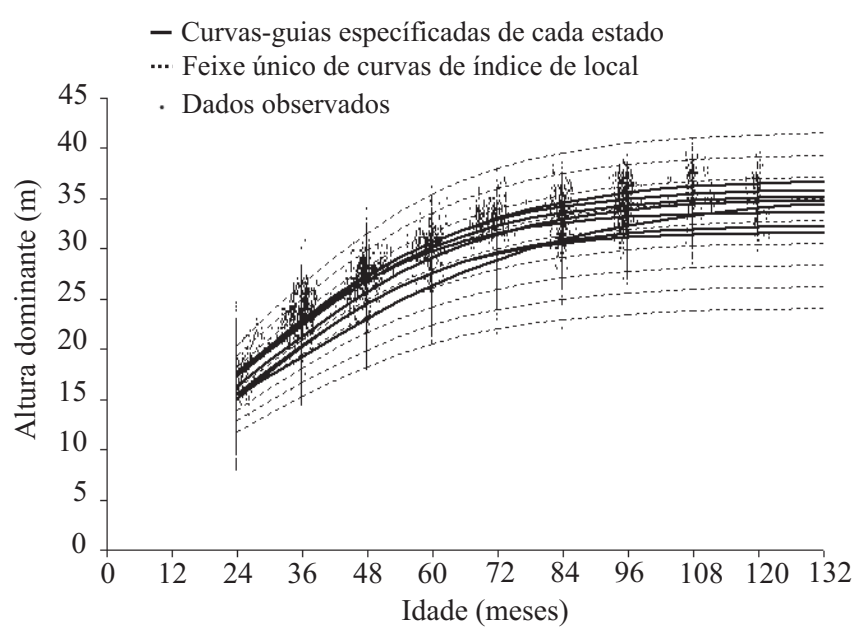

Figura 4. Dados observados de altura dominante, curvas-guia específicas de cada um dos oito estratos, e feixe único de curvas de índice de local, com uso de expressão única.

\section{Conclusões}

1. O procedimento proposto, para estratificação de florestas clonais de eucalipto e construção de curvas de índices de local, é eficiente e agrupa clones com tendências semelhantes de crescimento em altura dominante.

2. A estratificação realizada por meio do procedimento proposto resulta em estimativas de volume mais precisas, próximo à idade de corte.

3. A estimativa de índice de local, para clones que possuem menos de três medições, pode ser feita a partir das informações de altura dominante, diâmetro médio, área basal e volume, obtidas do inventário florestal contínuo de outros clones.

\section{Referências}

ABREU, V.M.N.; SILVA, M. de A. e; CRUZ, C.D.; FIGUEIREDO, E.A.P. de; ABREU, P.G. de. Desempenho e predição de híbridos e análises de agrupamento de características de matrizes de frangos de corte. Revista Brasileira de Zootecnia, v.31, p.617-626, 2002.

ALBUQUERQUE, M.A. de; FERREIRA, R.L.C.; SILVA, J.A.A. da; SANTOS, E. de S.; STOSIC, B.; SOUZA, A.L. de. Estabilidade em análise de agrupamento: estudo de caso em ciência florestal. Revista Árvore, v.30, p.257-265, 2006.

CAMPOS, J.C.C.; LEITE, H.G. Mensuração florestal: perguntas e respostas. 2.ed. Viçosa: UFV, 2006. 470p.
CLUTTER, J.L. Compatible growth and yield for loblolly pine. Forest Science, v.9, p.354-371, 1963.

CRUZ, C.D.; REGAZZI, A.J.; CARNEIRO, P.C.S. Modelos biométricos aplicados ao melhoramento genético. Viçosa: UFV, 2004. v.1. 480p.

CURTIS, R.O.; MARSHALL, D.D. Permanent-plot procedures for silvicultural and yield research. Portland: U.S. Department of Agriculture, 2005. 86p.

DAVIS, L.S.; JOHNSON, K.M. Forest management. New York: McGraw-Hill Book Company, 1987. 790p.

DIAS, A.N.; LEITE, H.G.; CAMPOS, J.C.C.; COUTO, L.; CARVALHO, F.C. de. Emprego de um modelo de crescimento e produção em povoamentos desbastados de eucalipto. Revista Árvore, v.29, p.731-739, 2005a.

DIAS, A.N.; LEITE, H.G.; NOGUEIRA, G.S.; RODRIGUES, F.L. Avaliação de métodos de ajuste de curvas de índices de local em povoamentos de eucalipto desbastados. Revista Árvore, v.29, p.741-747, 2005b.

DRAPER, N.R.; SMITH, H. Applied regression analysis. $3^{\text {rd }}$ ed. New York: John Willey \& Sons, 1998. 706p.

HEGER, L. Effect of index age on the precision of site index. Canadian Journal of Forest Research, v.3, p.1-6, 1973.

MARTINS, S.V.; SILVIA, N.R.S.; SOUZA, A.L. de; MEIRA NETO, J.A.A. Distribuição de espécies arbóreas em um gradiente topográfico de floresta estacional semidecidual em Viçosa, MG. Scientia Forestalis, v.64, p.172-181, 2003.

PARRESOL, B.R.; VISSAGE, J.S. White pine site index for the Southern forest survey. Asheville: U.S. Department of Agriculture, 1998. 8p.

SANTOS, G.A. dos; XAVIER, A.; LEITE, H.G. Desempenho silvicultural de clones de Eucalyptus grandis em relação às árvores matrizes. Revista Árvore, v.30, p.737-747, 2006.

SANTOS, J.H. da S.; FERREIRA, R.L.C.; SILVA, J.A.A. da; SOUZA, A.L. de; SANTOS, E. de S.; MEUNIER, I.M.J. Distinção de grupos ecológicos de espécies florestais por meio de técnicas multivariadas. Revista Árvore, v.28, p.387-396, 2004.

SCHUMACHER, F.X. A new growth curve and its application to timber studies. Journal of Forestry, v.37, p.819-820, 1939.

TONINI, H.; SCHNEIDER, P.R.; FINGER, C.A.G. Crescimento de clones de Eucalyptus saligna Smith, na Depressão Central e Serra do Sudeste, Rio Grande do Sul. Ciência Florestal, v.14, p.61-77, 2004.

VALDEZ-LAZALDE, J.R.; LYNCH, T.B. Merchantable and total volume equations for thinned stands of Patula Pine in Puebla, México. Agrociencia, v.34, p.747-758, 2000.

VANCLAY, J.K.; SKOVSGAARD, J.P.; PILEGAARD HANSEN, C. Assessing the quality of permanent sample plot databases for growth modelling in forest plantations. Forest Ecology and Management, v.71, p.177-186, 1995.

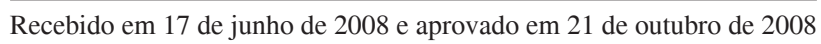


